Groups Geom. Dyn. 7 (2013), 419-442

DOI $10.4171 / \mathrm{GGD} / 188$
Journal of Noncommutative Geometry

(C) European Mathematical Society

\title{
Cubulating rhombus groups
}

\author{
David Janzen ${ }^{1}$ and Daniel T. Wise ${ }^{2}$
}

\begin{abstract}
We study CAT(0) 2-complexes all of whose polygons are Euclidean rhombi. We describe walls in these complexes, and examine the dual CAT(0) cube complex. Our viewpoint relates Sageev's dual cube complexes to de Bruijn's approach to the Penrose tilings.
\end{abstract}

Mathematics Subject Classification (2010). 20F67.

Keywords. CAT(0) cube complexes, Penrose tilings.

\section{Introduction}

A square complex $X$ is a cell complex obtained by attaching squares to a graph by identifying the bounding edges with edges in the graph. The square complex $X$ is nonpositively curved if for each $x \in X^{0}$, the graph link $(x)$ does not contain a cycle of length $\leq 3$. We recall that $\operatorname{link}(x)$ is roughly the " $\epsilon$-sphere" about $x$ in $X$ and contains a vertex for each end of a 1-cell at $x$ and contains an edge for each corner of a 2-cell at $x$. For example, the usual cell-complex associated to a cartesian product $A \times B$ of two graphs is a nonpositively curved square complex.

Nonpositively curved square complexes are the simplest type of $C(4)-T(4)$ small cancellation complexes, and have been studied well before Gromov's geometric group theory revolution, which amongst an enormous number of richly innovative ideas, introduced higher-dimensional nonpositively curved cube complexes. These have been playing an increasingly central role in the field, as they naturally encode information about the codimension-1 subgroups of a group.

The purpose of this paper is to examine a 2-dimensional generalization of nonpositively curved square complexes: Namely, piecewise Euclidean complexes that are built from parallelograms instead of squares. The link condition corresponds naturally to a $2 \pi$ lower bound on the angle sum of corners associated to any cycle in each link $(x)$. As parallelograms can be scaled without affecting the angles to obtain rhombi, we are naturally led to focus on nonpositively curved rhombus complexes. We describe several sources of examples in Section 2.

\footnotetext{
$1 \uparrow$ Supported by an NSERC Canada Graduate Scholarship.

$2+$ Supported by NSERC.
} 
Our main result is:

Theorem 1.1. Let $X$ be a nonpositively curved rhombus complex. Then $\pi_{1} X$ acts properly and freely on a $\mathrm{CAT}(0)$ cube complex.

At the expense of added dimension, Theorem 1.1 places rhombus complexes structurally together with the nonpositively curved square complexes that they generalize. This result joins a larger body of work attesting to the ubiquity of group actions on CAT( 0 ) cube complexes. Theorem 1.1 is proven by producing a sufficient collection of walls in $\widetilde{X}$ and then considering Sageev's dual cube complex. The walls in the universal cover of a rhombus complex are described in Section 3, properties of Sageev's dual cube complex are reviewed in Section 4, and the main result is proven in Section 5.

We were unable to determine whether $\pi_{1} X$ always acts freely on a finite dimensional CAT(0) cube complex, but we describe some partial results towards finite dimensionality in Section 6. In particular, we show that the cube complex associated to $\tilde{X}$ is finite dimensional when $X$ is a rhombus complex arising from a compact "even complex" which is a nonpositively curved 2-complex whose 2-cells are regular Euclidean polygons with an even number of sides. In this case our results show that the cube complex associated to $\widetilde{X}$ has a "cosparse" structure very similar to the type of cube complex that often arises from a group that is hyperbolic relative to abelian subgroups. Verification of this finiteness property requires a very explicit understanding of systems of pairwise crossing walls that are explored in Sections 7 and 8.

Drawn towards the most famous rhombus complexes - the Penrose tilings, we find that our method is synchronized with de Bruijn's beautiful treatment of the Penrose tilings, which remarkably anticipates Sageev's construction. This interesting connection is discussed in Section 9.

One of the striking unsettled problems about a group $G$ acting cocompactly on a $\mathrm{CAT}(0)$ space is whether the following always holds:

Either $G$ is word-hyperbolic or $G$ contains a copy of $\mathbb{Z}^{2}$.

This problem is even open when $G=\pi_{1} X$ where $X$ is a compact nonpositively curved square complex, and we refer the reader to [Wis05] for some limited partial positive results in this case.

For a cocompact CAT(0) space, there is a dichotomy [BH99] between wordhyperbolicity and the existence of a flat plane, and so the above assertion asks whether a flat plane in $\tilde{X}$ implies the existence of a flat plane that is periodic in the sense that its stabilizer contains $\mathbb{Z}^{2}$. As Gromov noted, the existence of aperiodic sets of tiles, like the Penrose rhombi, suggest that there are counterexamples to the above italicized assertion. We agree. However, at the end of Section 9 we describe the natural way of producing a compact (nonpositively curved) rhombus complex $X$ from the Penrose rhombi. Curiously, we find that $\pi_{1} X$ is always word-hyperbolic. 


\section{Rhombus complexes}

Definition 2.1. A rhombus complex is a 2-complex whose 2-cells are rhombi in the sense that the attaching maps are combinatorial paths of length 4 and there are angle assignments to the edges in the link of each 0 -cell of the complex, such that adjacent corners of each 2-cell have complementary angles.

Accordingly, there is a piecewise Euclidean structure where we can declare each 1-cell to have unit length, and declare each 2-cell to be a unit side-length Euclidean rhombus. However, the CAT(0) metric plays little role here, and our arguments primarily utilize angles and the combinatorial Gauss-Bonnet theorem.

For the purposes of this paper, we assume that all the angles assigned are strictly between 0 and $\pi$. This will rule out pathological behavior illustrated in Figure 1. As will be shown later, in a rhombus complex with angles strictly between 0 and $\pi$, walls cannot self-intersect nor can pairs of walls form bigons.
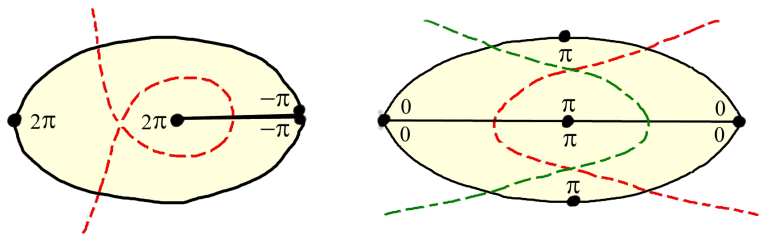

Figure 1. On the left is a self-intersecting wall in a rhombus complex where negative angles are allowed. On the right is a pair of walls forming a bigon in a rhombus complex where zero angles are allowed.

2.1. An explicit example. We now describe a rhombus complex $X$ that is formed by attaching six rhombi to a graph. The data for $X$ is illustrated in Figure 2. The
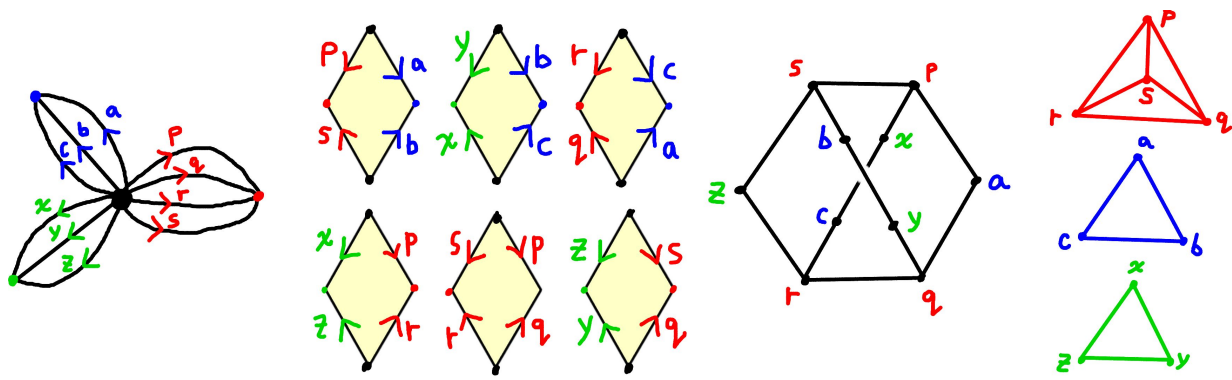

Figure 2. Attach the 6 rhombi above to the graph at the left to obtain a rhombus complex whose links are at the right.

1-skeleton $X^{1}$ is illustrated on the left, and the six rhombi are illustrated in the center. The links of the four 0 -cells of $X$ are illustrated on the right. The rhombi have angles 
$\frac{2 \pi}{3}, \frac{\pi}{3}$ at the coloured and black vertices respectively. The nonpositive curvature condition is satisfied since the corresponding black and colored links have girths 6 and 3 .

2.2. Triangles of groups. We now describe an example arising from Stallings nonpositively curved triangles of groups [Sta91]. Let $T$ be a triangle of groups with vertex groups $V_{1}, V_{2}, V_{3}$ and edge groups $E_{12}, E_{23}, E_{31}$ and face group $F$. Suppose the following hold:

(1) $T$ is nonpositively curved,

(2) $E_{12}, E_{31}$ together generate $V_{1}$,

(3) $\frac{\pi}{2}=\operatorname{Angle}\left(E_{12} *_{F} E_{31} \rightarrow V_{1}\right)$,

(4) $\left[E_{12}: F\right]=2=\left[E_{31}: F\right]$.

Then the universal cover $\tilde{T}$ gives rise to a rhombus complex whose two angles are twice the angles of $T$ at $V_{2}$ and $V_{3}$. Note that, if necessary, one can first increase these angles in $T$ so that they are complementary. As in Figure 3, one "unsubdivides" to obtain a rhombus for each copy of the vertex $\widetilde{V}_{1}$ in $\widetilde{T}$. The group $\pi_{1} T$ acts properly and cocompactly on this rhombus complex.
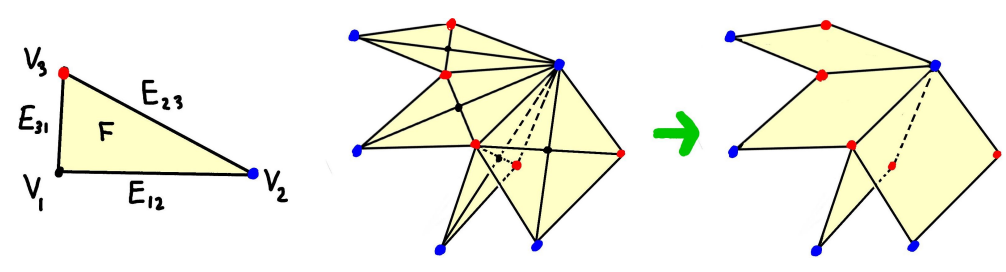

Figure 3. On the left is a nonpositively curved triangle $T$ of groups with $\mathbb{Z}_{2} \subset \mathbb{Z}_{2}^{2} \supset \mathbb{Z}_{2}$ at the right-angled corner. On the right we partially illustrate how to "unsubdivide" $\widetilde{T}$ to obtain a rhombus complex.

For instance, the above specifications apply to a nonpositively curved triangle of groups whenever the face group is trivial, there is a $\mathbb{Z}_{2} \times \mathbb{Z}_{2}$ vertex group, and its two edge groups are distinct copies of $\mathbb{Z}_{2}$.

\subsection{Paired polygons}

Definition 2.2. A convex Euclidean polygon with no $\pi$ angles at corners is a paired polygon if every edge has been paired with exactly one other edge parallel to it. Note that paired polygons have an even number of edges.

Lemma 2.3. Paired polygons can be subdivided into parallelograms.

Proof. Begin by choosing a pair of parallel edges. These edges determine a top and a bottom of the polygon. Translating the top toward the bottom as illustrated in Figure 4 


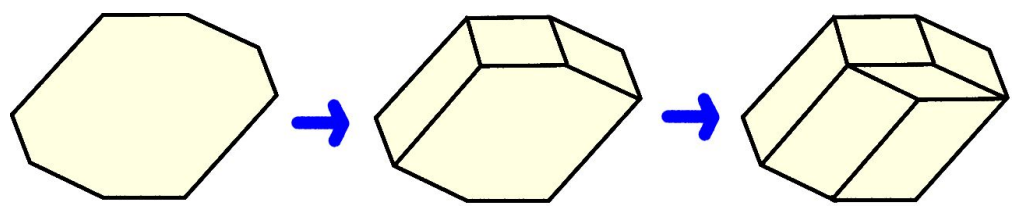

Figure 4. By induction, a paired polygon can be subdivided to obtain a parallelogram complex.

one obtains a complex containing some parallelograms and a new paired polygon with two fewer sides than the original. Continuing in this fashion one eventually obtains a complex all of whose polygons are parallelograms.

Definition 2.4. A paired 2-complex $X$ is a piecewise Euclidean 2-complex whose 2 -cells are all paired polygons. More specifically, an even 2-complex has the requirement that all 2-cells are regular polygons with an even number of sides.

A simple consequence of Lemma 2.3 is the following:

Corollary 2.5. Every nonpositively curved paired 2-complex has a subdivision that is a rhombus complex.

Corollary 2.5 implies that all aspherical surfaces are homeomorphic to rhombus complexes. This applies to the standard 2-complex of the usual one-relator presentations of surface groups. It is well known that such surfaces are fundamental groups of nonpositively curved square complexes. However, the rhombus complex obtained by Corollary 2.5 is different. For instance, it shows that there are cocompact rhombus complexes whose dual cube complexes have arbitrarily high dimension. The wallspaces obtained are the same as those that arise from small cancellation theory in [Wis04].

A beautiful source of even CAT( 0 ) 2-complexes arise from 2-dimensional Coxeter groups. These are the Coxeter groups whose presentations do not contain a subpresentation consisting of a spherical triangle group. The universal cover of the standard 2-complex yields a 2-complex $\widetilde{X}$ with a $G$-action: It is obtained by identifying bigons to single edges, and identifying repeated 2-cells to a single 2-cell in the usual fashion.

We note that the subdivision of Lemma 2.3 is not consistent with the dihedral group action on the polygon. Accordingly, if the action of $G$ on a CAT(0) 2-complex is not free, and more specifically, has nontrivial 2-cell automorphisms, then the rhombus subdivision of Lemma 2.3 might not be preserved by the action. However, we emphasize that the wallspace structure within a paired polygon obtained by subdivision using Lemma 2.3 does not depend upon the choice of subdivision. This is because walls are associated to paired edges.

As Coxeter groups are virtually torsion-free, we do still obtain an action on the rhombus complex. 
Corollary 2.6. Let $G$ be a 2-dimensional Coxeter group, and let $G^{\prime}$ be a finite index torsion-free subgroup of $G$. Then $G^{\prime}$ acts properly and cocompactly on a CAT(0) rhombus complex. Moreover, $G$ acts properly on the CAT(0) cube complex dual to $\tilde{X}$.

We emphasize that Corollary 2.6 is only a special case of a result of Niblo-Reeves [NR03] who showed that every finitely generated Coxeter group acts properly on a finite dimensional CAT(0) cube complex. The action we obtain is the same.

\section{The walls in a rhombus complex}

Definition 3.1. Let $X$ be a rhombus complex. A midcube of a rhombus is one of the two line segments passing through the center starting and ending on one pair of sides and parallel to the other pair of sides. An immersed wall in $X$ is a graph whose intersection with each rhombus is either empty or a midcube of the rhombus. There is a graph $\Gamma_{X}$ whose vertices are midcubes of 1-cells of $\tilde{X}$ and whose edges are midcubes of 2-cells of $\tilde{X}$. Note that the components of the graph $\Gamma_{X}$ are the immersed walls of the rhombus complex $\tilde{X}$. We will later prove that these immersed walls actually embed and then refer to the immersed walls simply as walls.

3.1. Review of combinatorial Gauss-Bonnet. We begin by recalling the combinatorial Gauss-Bonnet theorem, a proof of which can be found in [MW02]. Let D be a disc diagram with a real number called an angle assigned to each corner of each 2 -cell in the diagram. For a 0 -cell $v$ in the diagram, one defines $\operatorname{Corners}(v)$ to be the set of corners of 2-cells incident with $v$. The curvature of a 0 -cell $v$ in the diagram is then:

$$
\kappa(v)=2 \pi-\pi \chi(\operatorname{link}(v))-\sum_{c \in \operatorname{Corners}(v)} \varangle(c)
$$

Similarly, Corners $(f)$ denotes the set of corners at a 2-cell $f$ and the curvature of $f$ is:

$$
\kappa(f)=(|\partial f|-2) \pi-\sum_{c \in \operatorname{Corners}(f)} \varangle(c)
$$

For rhombus complexes this simplifies to $\kappa(f)=2 \pi-\sum_{c \in \operatorname{Corners}(f)} \varangle(c)=0$.

Having explained how to distribute the Euler characteristic of the disc diagram $D$ as "curvature" concentrated at 0 -cells and 2-cells, we now state the combinatorial Gauss-Bonnet theorem which recovers the Euler characteristic.

Theorem 3.2 (Combinatorial Gauss-Bonnet).

$$
2 \pi=\sum_{v \in 0 \text {-cells }(X)} \kappa(v)+\sum_{f \in 2 \text {-cells }(X)} \kappa(f) .
$$


3.2. Curvature shifting along the boundary of rhombus diagrams. A corner of a rhombus in a disc diagram $D$ is a 0 -cell, $v$, in $\partial D$ such that link $(v)$ consists of two vertices joined by an edge.

If $v \in \partial D$ is a 0 -cell on the boundary of a nonsingular disc diagram $D$, we assign a cyclic ordering to the corners, $c_{1}, c_{2}, \ldots, c_{d}$, of 2-cells incident with $v$. This induces an ordering of their angles $\varangle_{1}, \ldots, \varangle_{d}$. We then have $\kappa(v)=\pi-\sum_{i=1}^{d} \varangle_{i}$.

We define $\bar{\kappa}(v)$ implicitly by the formula:

$$
\kappa(v)=\left(\frac{\pi}{2}-\varangle_{1}\right)+\bar{\kappa}(v)+\left(\frac{\pi}{2}-\varangle_{d}\right)
$$

We will use the notation $\bar{\kappa}(v)=\left(\frac{\pi}{2}-\varangle_{1}\right)$ and $\vec{\kappa}(v)=\left(\frac{\pi}{2}-\varangle_{d}\right)$. Note that $\bar{\kappa}(v)=-\sum_{i=2}^{d-1} \varangle_{i}$ when $d \geq 2$ and hence $\bar{\kappa}(v)=0$ when $d=2$. We emphasize that in the degenerate $d=1$ case where $v$ is a corner, we have $\bar{\kappa}(v)=\varangle(v)$.
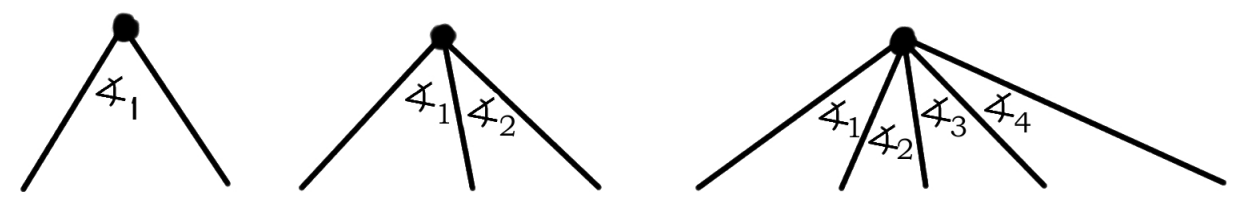

Figure 5. In the first case $\bar{\kappa}(v)=\varangle_{1}$, while in the second case $\bar{\kappa}(v)=0$ and in the third $\bar{\kappa}(v)=-\sum_{i=2}^{d-1} \varangle_{i}=-\left(\varangle_{2}+\varangle_{3}\right)$.

Lemma 3.3. Let $D$ be a nonsingular disc diagram that is a nonpositively curved rhombus complex. Then $\sum_{c \in \operatorname{Corners}(D)} \varangle(c) \geq 2 \pi$. (Moreover, the sum equals $2 \pi$ iff $D$ is a "grid".)

Proof. Using Theorem 3.2 and the fact that $\kappa(f)=0$ for every 2-cell $f$ in a rhombus complex, we have $2 \pi=\sum \kappa(v)+\sum \kappa(f)=\sum \kappa(v)=\sum_{v \in \partial D} \kappa(v)+$ $\sum_{v \notin \partial D} \kappa(v)$. Since $\kappa(v) \leq 0$ whenever $v \notin \partial D$, we thus have $2 \pi \leq \sum_{v \in \partial D} \kappa(v)$.

Let $v_{0}, v_{1}, \ldots, v_{n}=v_{0}$ denote the 0 -cells of $\partial D$ in cyclic order about $D$. Note that when $v$ is not a corner $\kappa(v)=\bar{\kappa}(v)+\bar{\kappa}(v)+\vec{\kappa}(v)$, but when $v$ is a corner then $\kappa(v)=\bar{\kappa}(v)+\bar{\kappa}(v)+\vec{\kappa}(v)=\left(\frac{\pi}{2}-\varangle(v)\right)+\varangle(v)+\left(\frac{\pi}{2}-\varangle(v)\right)=\pi-\varangle(v)$.

Now for any 0 -cells $v_{i}, v_{i-1}$ on the same rhombus, one has that $\bar{\kappa}\left(v_{i}\right)+\vec{\kappa}\left(v_{i-1}\right)=$ 0 since the angle sum of adjacent angles on the same rhombus is $\pi$. We therefore obtain: $\sum_{v \in \partial D} \kappa(v)=\sum_{i=1}^{n} \kappa\left(v_{i}\right)=\sum_{i=1}^{n}\left(\bar{\kappa}\left(v_{i}\right)+\bar{\kappa}\left(v_{i}\right)+\vec{\kappa}\left(v_{i}\right)\right)=$ $\sum_{i=1}^{n}\left(\bar{\kappa}\left(v_{i}\right)+\vec{\kappa}\left(v_{i-1}\right)+\bar{\kappa}\left(v_{i}\right)\right)=\sum_{i=1}^{n} \bar{\kappa}\left(v_{i}\right) \leq \sum_{v_{i} \in \operatorname{Corners}(D)} \varangle\left(v_{i}\right)$ where the last inequality holds since $\bar{\kappa}(v) \leq 0$ unless $v$ is a corner in $D$.

Lemma 3.4. Let $D \rightarrow \tilde{X}$ be a minimal area disc diagram in a rhombus complex. Then either $D$ is a single 0 -cell, $D$ is an arc, or D has at least three corners and/or spurs. 
Proof. The singular case follows easily from the nonsingular case and the fact that a spur can "hide" at most one corner. When $D \rightarrow \tilde{X}$ is a nonsingular minimal area disc diagram, the result follows from Lemma 3.3 since each $\varangle \leq \pi$ and $2 \pi \leq$ $\sum_{v \in \operatorname{Corners}(D)} \varangle(v)$.

3.3. Geometry of the walls of $\tilde{\boldsymbol{X}}$. Lemma 3.4 allows us to draw useful conclusions about immersed walls. In order to facilitate its application, we observe that if $X^{\prime}$ is the obvious subdivision of $\widetilde{X}$ obtained by subdividing each 1-cell into two 1-cells and each rhombus into four rhombi, then $X^{\prime}$ is a CAT(0) rhombus complex whenever $\tilde{X}$ is. The immersed walls of $\tilde{X}$ map to the 1 -skeleton of $X^{\prime}$. The subdivision $X^{\prime}$ of $\tilde{X}$ enables the following consequence of Lemma 3.4:

Theorem 3.5. (0) The immersed walls in $\tilde{X}$ are trees.

(1) The immersed walls in $\tilde{X}$ embed in $\tilde{X}$.

(1 $\left.\frac{1}{2}\right)$ Adjacent 1 -cells of $\tilde{X}$ cannot be dual to the same wall.

(2) If $w_{1}, w_{2}$ are distinct immersed walls in $\tilde{X}$ then $w_{1} \cap w_{2}$ contains at most one point.

Proof. Immersed walls cannot: (0) form closed loops, (1) self-intersect, or (2) form bigons. Each case would lead to a disc diagram in the rhombus complex $X^{\prime}$ with zero, one or two corners respectively thereby violating Lemma 3.4.

Let us now consider $\left(1 \frac{1}{2}\right)$. Suppose adjacent edges $e_{1}, e_{2}$ are dual to the same wall $w$. By part (1) we know that $e_{1}, e_{2}$ do not meet at the corner of a single rhombus. Observe that there is a closed path in $X^{\prime}$ of the form $\alpha_{1} \sigma \alpha_{2}$ where $\sigma$ is a path in $w$ and $\alpha_{1}, \alpha_{2}$ are halves of $e_{1}, e_{2}$. A disc diagram $D$ for $\alpha_{1} \sigma \alpha_{2}$ has at most two corners since there is no corner at $\alpha_{1} \alpha_{2}$.

Remark 3.6. We note that Theorem $3.5(0)$ and (1) continue to hold for rhombus complexes with degenerate rhombi whose angles are 0 and $\pi$. We have not pursued this generalization as the crucial point enabling the consequences we obtain is contained in Theorem 3.5 (2).

Definition 3.7. Given an immersed wall $w$ we define its carrier $N=N(w)$ to be the subcomplex of $\widetilde{X}$ consisting of all rhombi whose intersection with $w$ is nonempty. Observe that $w \subset N$ and that there is an immersion from $N$ to $\widetilde{X}$. We also note that there is a degenerate case, where $w$ is a wall consisting of a single point at the center of a 1-cell, in which case, this closed 1-cell is its carrier. In contrast to Theorem 3.5 (2) it is possible that $N\left(w_{1}\right) \cap N\left(w_{2}\right)$ is disconnected as in Figure 6.

Theorem 3.8. Let $\tilde{X}$ be the universal cover of a rhombus complex $X$. Then the carrier $N(w)$ of the wall $w$ is isomorphic to $w \times[-1,1]$ and embeds in $\tilde{X}$. 
Proof. Consider a combinatorial map $w \times[-1,1] \rightarrow \tilde{X}$ extending the inclusion $w \hookrightarrow \tilde{X}$. If it is not injective then there are two 0 -cells in $w \times[-1,1]$ mapping to the same 0 -cell in $\tilde{X}$. This means that there are distinct 1-cells $e_{1}, e_{2}$ in $\tilde{X}$ which are dual to the same wall $w$ and adjacent. This contradicts Theorem $3.5\left(1 \frac{1}{2}\right)$.

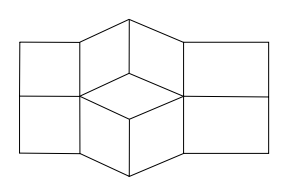

Figure 6. The intersection of two carriers need not be connected.

In fact, the carriers $N(w)$ actually embed isometrically in $\tilde{X}$ as we show next.

We say that two points $p, q$ are separated by a wall $W$ if they lie in distinct parts of its partition. We use the notation \# $(p, q)$ to denote the total number of walls separating $p, q$. The following lemma relates the length of a geodesic to the number of walls separating its endpoints.

Lemma 3.9. Let $p, q$ be 0 -cells in $\tilde{X}$. Then $d_{\tilde{X}}(p, q)=\#(p, q)$.

Proof. We emphasize, that Lemma 3.9 and its proof focus on combinatorial geodesics, which are minimal length edge-paths connecting 0-cells in the 1-skeleton. However, consideration of the smallest subcomplex containing a CAT( 0$)$-metric geodesic shows that the combinatorial length of a geodesic is approximately the same as the length of a CAT(0)-metric geodesic with the same endpoints.

Let $\gamma$ be a combinatorial geodesic in $\tilde{X}$ with endpoints $p$ and $q$. Using the facts that walls embed in $\widetilde{X}$ and that the carrier $N(w)$ of the wall $w$ is isomorphic to $w \times[-1,1]$, we observe that $d_{\tilde{X}}(p, q) \geq \#(p, q)$ because each wall separating $p$, $q$ must cross $\gamma$ at least once. We show that no wall crosses $\gamma$ at two distinct edges. Consequently there is exactly one crossing wall for each edge of $\gamma$.

Suppose there is a wall, $w$, that crosses the geodesic $\gamma$ in distinct edges $d, e$. The situation is represented in Figure 7.

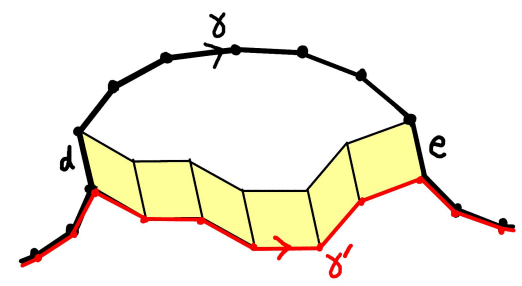

Figure 7. A wall is not double-crossed by a geodesic. 
Consider a ladder $L$ in the carrier of $w$ such that the first and last rungs of $L$ are the edges $d, e$. Note that the edges $d, e$ cannot be adjacent by Theorem $3.5\left(1 \frac{1}{2}\right)$. Let $\sigma$ denote the subpath of $\gamma$ between $d$ and $e$.

Let $r, r^{\prime}$ denote the rails of $L$ where $r$ denotes the rail with the same endpoints as $\sigma$. By Theorem 3.5 (2), there are no wall bigons, and so the edges of $r$ are dual to distinct walls. As the endpoints of $r$ lie on opposite sides of these distinct walls, the codimension-1 property implies that each such wall crosses $\sigma$. Thus $\left|r^{\prime}\right|=|r| \leq|\sigma|$.

Finally, we can substitute $r^{\prime}$ for $d \sigma e$ in $\gamma$ to obtain a new path $\gamma^{\prime}$ with $\left|\gamma^{\prime}\right|=|\gamma|-2$ and this contradicts our assumption that $\gamma$ is a geodesic.

Lemma 3.10. Let $\tilde{X}$ be the universal cover of a rhombus complex $X$. Then the carrier $N(w)$ of the wall $w$ embeds isometrically in $\tilde{X}$.

Proof. This follows from Lemma 3.9.

Theorem 3.11. Let $\gamma$ be a combinatorial straight path. Then if walls $w_{1}$ and $w_{2}$ are dual to distinct 1-cells of $\gamma$ then $w_{1} \cap w_{2}=\phi$.

Proof. This is essentially a consequence of Lemma 3.3. We consider a minimal area diagram between $w_{1}, w_{2}$ and $\gamma$, as partially illustrated in Figure 8 . Because $\gamma$ is straight, the angle between $w_{1}$ and $\gamma$ and the angle between $w_{2}$ and $\gamma$ are complementary, so their sum is $\pi$. Since the curvature at the remaining corner is less than $\pi$, the situation cannot happen.

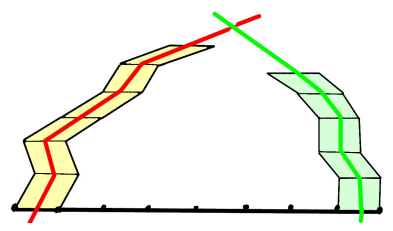

Figure 8. Walls dual to edges in a straight geodesic cannot cross.

Theorem 3.12 (Walls intersecting in convex subcomplexes). Let $C \subset \tilde{X}$ be a convex subcomplex. Let $w_{1}, w_{2}$ be walls dual to 1-cells of $C$. If $w_{1} \cap w_{2} \neq \phi$ then $\left(w_{1} \cap w_{2}\right) \subset C$.

Proof. Let $D$ be a minimal area diagram between $w_{1}, w_{2}$ and $C$. Because $C$ is convex and $D$ is minimal, $\partial D \cap C$ determines a subpath $\gamma$ with nonpositive curvature in $D$. This reduces to the same situation as in Figure 8.

We close this section with the following: 
Question 3.13. What kinds of generalizations are possible to higher-dimensional cases? Can analogous systems of walls be defined for nonpositively curved parallelepiped complexes?

\section{Background on dual cube complexes}

4.1. Wallspaces. "Spaces with walls" were introduced by Haglund and Paulin in [HP98] and we follow the treatment of their ideas given in [HW]. A wallspace is a space $\widetilde{X}$ with a collection $\mathcal{W}$ of walls which are subspaces $w \subset \widetilde{X}$ with the property that $\tilde{X}-w$ consists of two connected components called halfspaces.

The walls in Section 3 qualify because they satisfy the following criterion. The space $\tilde{X}$ is simply-connected, and $w$ has a neighborhood in $\tilde{X}$ (its carrier) that is homeomorphic to $w \times[-1,1]$ with $w$ corresponding to $w \times\{0\}$.

4.2. Sageev's construction. Sageev introduced a simple but powerful construction that yields an action of $G$ on a CAT(0) cube complex from an action of $G$ on a wallspace $\tilde{X}$ [Sag95]. The CAT(0) cube complex $C$ that is dual to this system of walls is defined as follows: The 0-cubes of $C$ consist of collections of halfspaces such that: for each wall, exactly one of its halfspaces lies in the collection; no two halfspaces in the collection are disjoint; and all but finitely many halfspaces contain a fixed basepoint $\tilde{x}$ of $\tilde{X}$. Two such 0 -cubes are joined by a 1-cube in $C$ if and only if the corresponding collections disagree in the choice of halfspace for exactly one wall. Having defined the 1-skeleton of $C$, higher cubes are added when their boundaries are present.

4.3. Finiteness properties of the $\boldsymbol{G}$-action on $\tilde{\boldsymbol{X}}$. When a group $G$ acts on a wallspace $\tilde{X}$ in a manner that permutes the halfspaces, there is an induced action of $G$ on the dual cube complex $C$. Finiteness properties of the $G$ action on $\tilde{X}$ lead to resulting finiteness properties of the $G$ action on $C$. The following result was obtained by Sageev in the hyperbolic case in [Sag97] and generalized to a relatively hyperbolic setting in [HW]. In particular, we note that a more elaborate version of Proposition 4.1 is given in [HW] that deals with more general relatively hyperbolic groups and provides a more specific picture of $C$.

Proposition 4.1. Let $G$ act properly and cocompactly on the wallspace $\tilde{X}$ and suppose each wall $w$ is quasi-isometrically embedded in $\tilde{X}$. Let $\tilde{Y}_{1}, \ldots, \widetilde{Y}_{r}$ be a collection of connected subspaces of $\tilde{X}$ called peripheries with the following property: For any collection $\left\{w_{j}\right\}_{j \in J}$ of pairwise crossing walls, there exists $g \in G$, and $k \in\{1, \ldots, r\}$ such that $w_{j}$ intersects $g \tilde{Y}_{k}$ for each $j \in J$.

Then there exists a compact subcomplex $K$ such that

$$
C(X)=G K \cup_{g \in G, 1 \leq k \leq r} g C\left(\tilde{Y}_{k}\right) .
$$


We work under the implicit assumption that each $w \cap g \widetilde{Y}_{k}$ is connected and so each $g \widetilde{Y}_{k}$ is itself a wallspace. This yields a dual cube complex $C\left(g \widetilde{Y}_{k}\right)$ and we note that $C\left(g \widetilde{Y}_{k}\right)=g C\left(\widetilde{Y}_{k}\right)$.

Remark 4.2. Sageev proved that when $G$ is word-hyperbolic, the dual cube complex $C(X)$ is $G$-cocompact. In this case the peripheries $\widetilde{Y}_{k}$ can be chosen to be sufficiently large finite balls.

If $G$ is hyperbolic relative to subgroups $P_{1}, \ldots, P_{r}$, then we aim to choose each $\tilde{Y}_{i}$ so that its stabilizer equals $P_{i}$. In this case, $f \widetilde{Y}_{i} \cap g \widetilde{Y}_{j}$ has uniformly finite diameter unless $\tilde{Y}_{i}=\tilde{Y}_{j}$ and $g^{-1} f \in \operatorname{Stabilizer}\left(\tilde{Y}_{i}\right)$.

This isolation of distinct peripheries, leads to an isolation of their dual cube complexes $C(g \widetilde{Y})$ from each other. More specifically, $f C\left(\tilde{Y}_{i}\right) \cap g C\left(\tilde{Y}_{j}\right) \subset G K$ unless $i=j$ and $g^{-1} f \in \operatorname{Stabilizer}\left(\tilde{Y}_{i}\right)$. As each $C\left(\tilde{Y}_{i}\right)$ is a convex subcomplex of $C(\tilde{X})$, this isolation, leads to a very specific picture of $C(\tilde{X})$, which essentially looks like a coarse copy of $G$, with coarsely disjoint copies of the $g C\left(\widetilde{Y}_{i}\right)$ attached along the corresponding $g P_{i}$.

In the special case where the $P_{i}$ are virtually abelian, the $C\left(\tilde{Y}_{i}\right)$ are quasi-isometric to $\mathbb{E}^{d_{i}}$ for some $d_{i}$, and then we have an even more precise picture of $C(X)$.

In parallel to the above cocompactness criterion there is a properness criterion for the action which we now state (see for example [HW]).

Proposition 4.3. Let $G$ be a finitely generated group with Cayley graph $\Gamma$. Suppose that $G$ acts properly on the wallspace $\tilde{X}$. Then $G$ acts properly on the dual cube complex $C$ provided the following holds for sequences of elements $p, q \in G$ : If $d_{\Gamma}(p, q) \rightarrow \infty$ then $\#(p, q) \rightarrow \infty$.

\section{Cubulating rhombus complexes}

In this section, we combine the properties of the walls with the dual cube complex construction.

Theorem 5.1. Let $X$ be a rhombus complex. Then $\pi_{1} X$ acts properly on the $\operatorname{CAT}(0)$ cube complex dual to the wallspace on $\widetilde{X}$.

Proof. The criterion of Proposition 4.3 holds for rhombus complexes since one has $d_{\tilde{X}}(p, q)=\#(p, q)$ as shown in Lemma 3.9.

Corollary 5.2. Let $X$ be a compact rhombus complex and let $w$ be a wall in $\tilde{X}$. Then Stabilizer $(w)$ quasi-isometrically embeds in $\pi_{1} X$.

Proof. This follows from the fact that carriers isometrically embed as proved in Lemma 3.10. 
Corollary 5.3. If $X$ is a compact rhombus complex such that $\pi_{1} X$ is word-hyperbolic, then the dual cube complex is finite dimensional and the group action is proper and cocompact.

Proof. As walls quasi-isometrically embed and $G$ is word-hyperbolic, we obtain that carriers are $k$-quasiconvex for some $k$. Quasiconvexity of carriers gives a cocompact group action, and properness was obtained in Theorem 5.1. From cocompactness we get finite dimensionality.

If $X$ is a compact rhombus complex, we do not know whether or not the cube complex associated to $\widetilde{X}$ is always finite dimensional. Sageev proved that this is the case when $\pi_{1} X$ is word-hyperbolic and this was generalized in [HW] to a relatively hyperbolic setting - but these are highly controlled special cases.

Conjecture 5.4. Let $X$ be a compact rhombus complex. Then the dual cube complex is finite dimensional. In particular, there is a uniform bound on the cardinality of any collection of pairwise crossing walls in $\widetilde{X}$.

A possible approach to Conjecture 5.4 is to generalize Theorem 8.1, which we prove in Section 8, and then apply Proposition 4.1.

When $X$ is not compact, there are examples where the dual cube complex is not finite dimensional. However, the reader can establish the following amusing point:

Example 5.5. Let $X$ be a compact rhombus complex. Suppose $X$ has an alternate piecewise Euclidean structure with the same angles, but where each polygon is a parallelogram that is not a rhombus. Then the dual cube complex is finite dimensional.

Remark 5.6. In Figure 9, an example is given where carriers are not $k$-quasiconvex for any $k$. (As noted in the proof of Corollary 5.3 such examples are necessarily not word-hyperbolic.) In this example, the group action on the complex is not cocompact. Are there cocompact examples where the carriers are not $k$-quasiconvex for any $k$ ?

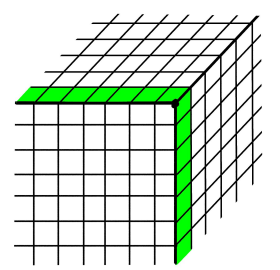

Figure 9. The rhombus complex obtained by gluing together three stretched quarter-planes provides an example where each wall carrier isometrically embeds (Lemma 3.10) but is not $k$-quasiconvex for any $k$. 


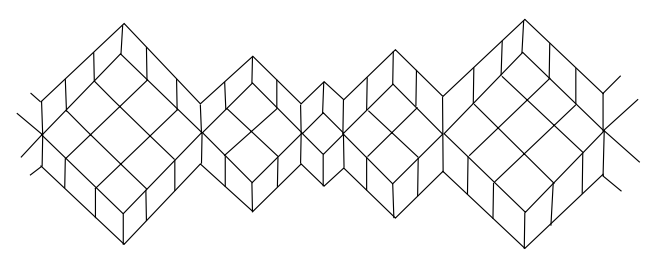

Figure 10. Carriers that diverge arbitrarily much.

In [GS90], Gersten and Short proved that $C(4)-T(4)$ groups are automatic, and this was later generalized from compact nonpositively curved square complexes to compact nonpositively curved cube complexes by Niblo-Reeves in [NR98].

Problem 5.7. Let $X$ be a compact rhombus complex. Is $\pi_{1} X$ (bi)automatic?

\section{Rhombus complexes with isolated flats and restricted peripheral subspaces}

A flat $F$ in $\tilde{X}$ is an isometric copy of $\mathbb{E}^{2}$. ACAT(0) 2-complex has isolated flats if there is a constant $D$ such that if $F_{1}, F_{2}$ are distinct flats in $\widetilde{X}$ then diameter $\left(F_{1} \cap F_{2}\right) \leq D$. The rhombus complex $X$ has isolated flats if its universal cover does.

Example 6.1. Let $X$ be a nonpositively curved 2-complex whose 2-cells are regular Euclidean polygons with at least 5-sides. Then $\widetilde{X}$ has isolated flats.

It is straightforward to prove the following which was initially observed in [Wis96] and developed further in [Hru04].

Proposition 6.2. Let $X$ be a compact $\mathrm{CAT}(0)$ 2-complex where $\tilde{X}$ has isolated flats. Then there are finitely many distinct orbits of flat planes in $\tilde{X}$ and each of them has virtually $\mathbb{Z}^{2}$ stabilizer. Moreover, $\pi_{1} X$ is hyperbolic relative to these stabilizers.

Theorem 5.1 combined with Proposition 4.1 then implies the relative cocompactness and in particular finite-dimensionality of the cube complex dual to the wallspace of $\widetilde{X}$ when it has isolated flats. Indeed the flats in $\widetilde{X}$ play the role of the peripheries $\widetilde{Y}$.

Simple examples show that the isolated flat property can fail for the even 2complexes of Definition 2.4: A notable example is where $X=A \times B$ and $A, B$ are graphs with $\chi(A), \chi(B)<0$.

However, when $X$ is even and compact, there are specific convex subcomplexes in $\tilde{X}$ whose orbits contain all possible flats. These are classified as follows:

(1) $(4,8,8)$-tilings of $\mathbb{E}^{2}$.

(2) $(6,6,6)$-tilings of $\mathbb{E}^{2}$. 
(3) $(4,6,12)$-tilings of $\mathbb{E}^{2}$.

(4) Convex subcomplexes $\widetilde{Q}$ that consist entirely of squares.

To see this, first note that there are finitely many eligible combinations of polygons, just based on considering sums of angles equal to $2 \pi$. Two consecutive polygons that are not both squares can only extend to a flat according to one of the tilings above, and moreover, they can do so uniquely if at all. The first three types of tilings can overlap with each other, or with a $\widetilde{Q}$ in at most a single tile.

There are finitely many orbits of flats of the first three types, and consequently, essentially following Proposition 6.2, each of these is periodic. The source of the difficulty in obtaining a periodic flat from a square subcomplex $\widetilde{Q}$, is that there can be an infinitely rich array of such subcomplexes containing flats, and they can overlap with each other in infinite subcomplexes.

We are now ready to establish our main result modulo a key property established in Sections 7 and 8.

Theorem 6.3. Let $X$ be a compact even 2-complex. Then $\pi_{1} X$ acts properly and relatively cocompactly on a finite dimensional CAT(0) cube complex.

We emphasize that there is no restriction on the convex square subcomplexes of $\tilde{X}-$ as these have a transparent effect on the dual cube complex. However, in many natural cases, each convex square subcomplex of $\tilde{X}$ is actually a cartesian product of two trees.

Proof. By Theorem 8.1, each collection of pairwise crossing walls is either represented in a uniformly bounded small triangle, in a 1-cell or 2-cell, or in one of the three types of (irreducible) periodic flats described above. We therefore define the peripheries to equal the finitely many orbits of irreducible periodic flats in $\tilde{X}$, together with finitely many orbits of finite balls in $\tilde{X}$.

Theorem 4.1 now implies that the dual cube complex is cocompact relative to the dual cube complexes of the irreducible flats. We moreover observe that since large triangles are represented in unique irreducible periodic flats, the dual cube complex is sparse in the sense that it is quasi-isometric to a copy of $\pi_{1} X$ with isolated copies of $\mathbb{E}^{n}$ sticking out.

This is the natural situation occurring in Remark 4.2, when $\pi_{1} X$ is hyperbolic relative to the stabilizers of irreducible periodic flats, however our explanation covers the general case.

Example 6.4. Any two-dimensional Coxeter group, that is, a Coxeter group whose Coxeter complex $\tilde{X}$ is a CAT(0) 2-complex, fits into the above framework. Its Euclidean triangle subgroups are associated to some of the flats. Its other flats are contained in subcomplexes $\widetilde{Q}_{i}$ associated to subcoxeter groups of the following form:

$$
\left\langle a_{i}: i \in I, b_{j}: j \in J \mid a_{i}^{2}, b_{j}^{2},\left(a_{i} b_{j}\right)^{2}: i \in I, j \in J\right\rangle
$$


Indeed, consider a Tree $\times$ Tree subcomplex $\tilde{Y}$ in $\tilde{X}$. Each of its walls is associated to a generator, and hence each vertical wall associated to some $a_{i}$ crosses each horizontal wall associated to some $b_{j}$ in a square 2-cell associated to a $\left(a_{i} b_{j}\right)^{2}$ relator. There are no relators of the form $\left(a_{i} a_{k}\right)^{m_{i k}}$ or $\left(b_{k} b_{j}\right)^{m_{k j}}$ since this would violate the 2dimensionality of $\widetilde{Q}$.

\section{Maximal triangles}

Lemma 7.1. Let $\tilde{X}$ be a wallspace, with the property that each wall is a tree, and for each pair of walls $w \neq w^{\prime}$, either $w \cap w^{\prime}=\emptyset$ or $w \cap w^{\prime}$ is a singleton. Assume that there are no infinite dimensional cubes - which in particular holds if the dual cube complex is finite dimensional. Then for each collection of pairwise crossing walls in $\tilde{X}$, there exists a triangle $T$ formed from (segments of) three of them, such that each wall in the collection intersects the triangle.

We note that if each edge of each wall is crossed by some other wall, then the "tree" hypothesis is superfluous as it follows from "no bigons".

Proof. This holds for any wall segment triangle $T$ that is maximal in the sense that no other such triangle $T^{\prime}$ lies within $T$ so that two sides of $T^{\prime}$ are sub-sides of $T$.

Indeed, suppose $T$ is maximal and is bounded by $w_{1}, w_{2}, w_{3}$. Consider another wall $w$ in the pairwise crossing collection.

Let $v_{i}$ denote the intersection of $w_{i+1}, w_{i-1}$ with subscripts in $\mathbb{Z}_{3}$, and label the vertices clockwise around $T$. At each $v_{i}$ there are subtrees $\vec{w}_{i+1}$ of $w_{i+1}$ and $\bar{w}_{i-1}$ of $w_{i-1}$ leaving $T$. Assign these notations so that the former refers to the "clockwise branch" from the viewpoint of $T$ and the latter refers to the "counter-clockwise branch". We refer to Figure 11.
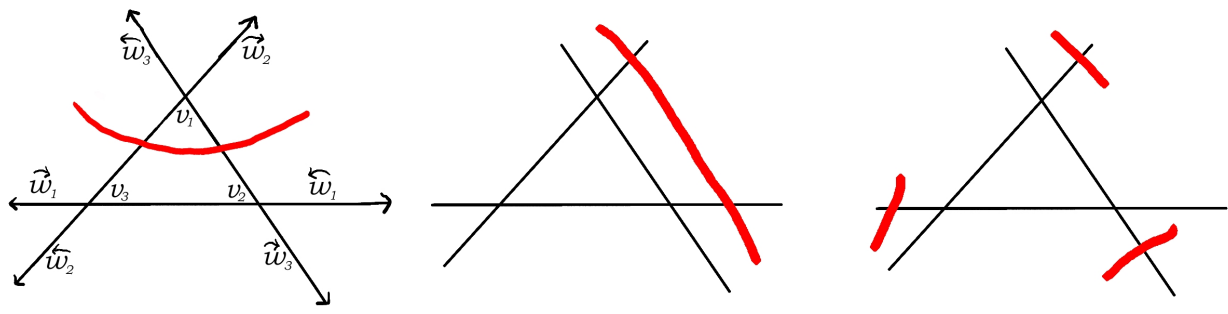

Figure 11. The next wall either hits the triangle, or forms a larger triangle containing the previous triangle. It is impossible to avoid these two possibilities as on the right.

We claim that $w$ must cross $T$ as on the left in Figure 11. If $w$ crosses some $\vec{w}_{i+1}, \bar{w}_{i}$ as in the middle, then we obtain a larger triangle which is impossible. The 
remaining case is partially illustrated on the right. Here $w$ crosses either each of $\vec{w}_{1}$, $\vec{w}_{2}, \vec{w}_{3}$, or each of $\overleftarrow{w}_{1}, \overleftarrow{w}_{2}, \overleftarrow{w}_{3}$.

Consideration of the ways these crossings can be connected in $w$ contradicts our hypotheses that $w$ is a tree and there are no bigons. Indeed, consider the $\vec{w}_{1}, \vec{w}_{2}$, $\vec{w}_{3}$ case. Observe that $w$ has nonempty intersection with the $\frac{1}{4}$-space of $w_{1}, w_{3}$ that contains $\vec{w}_{2}$, and $w$ also has nonempty intersection with its complementary $\frac{3}{4}$-space. However, $w$ is disjoint from the intersection of this $\frac{1}{4}$-space and $\frac{3}{4}$-space which equals $\bar{w}_{3} \cup \bar{v}_{1} v_{2} \cup \bar{w}_{1}$. This contradicts the connectivity of $w$.

\section{Representing wall triangles in compact even 2-complexes}

The goal of this section is to prove the following:

Theorem 8.1. Let $X$ be a compact nonpositively curved even 2-complex. There exists a collection of peripheries that are translates of finitely many flat planes and finitely many compact subcomplexes, such that any cube is represented in some periphery in the following sense: For each collection of pairwise crossing walls, there is a periphery such that each of these walls intersects the periphery.

Lemma 8.2. Let $X$ be a compact even 2-complex. For each $\delta$ there exists $L(\delta)$ such that for any $\delta$-thin triangle $T$ whose sides are geodesics in walls, each side of $T$ has length $\leq L(\delta)$.

Proof. Let $p$ be the maximal number of sides in a 2-cell of the compact complex $X$. Then the angle of intersection at crossing walls is bounded below by $\frac{2 \pi}{p}$. The $\operatorname{CAT}(0)$ inequality shows that a pair of crossing walls at some vertex can $\delta$-fellow travel for at most $\frac{\delta}{2 \sin \left(\frac{\pi}{p}\right)}$. Each side of $T$ thus has length at most $L(\delta)=\frac{\delta}{\sin \left(\frac{\pi}{p}\right)}$.

Lemma 8.3. There exists $f$ depending on the compact complex $X$ such that the following holds: Let $T$ be a wall triangle in $\widetilde{X}$. If one side of $T$ has length $\leq n$, then each side of $T$ has length $\leq f(n)$.

Proof. As in Lemma 8.2, the angle between walls is always $\geq \frac{2 \pi}{p}$. Observe that an isosceles triangle in $\mathbb{E}^{2}$ with base of length $\leq n$ and opposing angle $\geq \frac{2 \pi}{p}$ has sides of length $\leq \frac{n}{2 \sin \left(\frac{\pi}{p}\right)}$. We now prove the lemma, as it follows that one of the other two sides has length $\leq \frac{n}{2 \sin \left(\frac{\pi}{p}\right)}$ and letting $f(n)=n+\frac{n}{2 \sin \left(\frac{\pi}{p}\right)}$, the third side also has length $\leq f(n)$ by the triangle inequality.

Lemma 8.4. There exists $M=M(X)$ such that for any wall $w$ with carrier $N(w)$ within a reduced disc diagram $D \rightarrow X$, if the following holds then $N(w)$ has period dividing $M$. 
(1) Some 2-cell c of $N(w)$ is not a square.

(2) $N(w)$ has a flat open neighborhood (on at least one side).

Proof. Consideration of the cells around $c$ and a neighboring 2-cell, shows that at each stage it is impossible to have four squares around a vertex - instead, the configuration must be of the form: $(6,6,6),(4,8,8)$, or $(4,6,12)$. More specifically, the collection of 2-cells and orientations within the flat neighborhood of $N(w)$ is determined locally at each stage. As $X$ is finite, there are finitely many possibilities, so some configuration recurs. We let $M$ be the least common multiple of all possible recurrence rates.

The proof of the following is similar:

Lemma 8.5. There exists $R=R(X)$ such that for any wall $w$ in a reduced disc diagram $D \rightarrow X$, its $R$-neighborhood $\mathcal{N}_{R}(w)$ is contained in a periodic flat provided the following hold.

(1) $N(w)$ contains a 2-cell that is not a square,

(2) $\mathcal{N}_{R}(w)$ is "flat" in the sense that it isometrically embeds in $\mathbb{E}^{2}$, and

(3) $\mathcal{N}_{R}(w)$ is "wide" in the sense that it contains an isometric copy of $[0, R] \times[0, R]$.

Lemma 8.6. There exists $Q=Q(X)$ such that, for any geodesic (wall) triangle $T$ in $\widetilde{X}$, the number of negatively curved vertices in a disc diagram $D$ surrounded by $T$ is bounded by $Q$.

Proof. As there are finitely many angles arising at corners of 2-cells in the compact 2-complex $X$, we see that there is a smallest cycle $\sigma$ in any $\operatorname{link}(x)$ such that the angle sum $s$ of edges of $\sigma$ is strictly greater than $2 \pi$. In particular, let $-r<0$ denote this negative curvature that is smallest in absolute value arising in a disc diagram. Let $V$ denote the number of negatively curved internal vertices in $D$, and note that the only vertices in $\partial T$ with nonzero curvature are at the three corners of $T$. Apply Theorem 3.2 to $D$, we see that $2 \pi<-V r+3 \pi$, so $V<\frac{\pi}{r}$. We thus let $Q=\frac{\pi}{r}$.

Proof of Theorem 8.1. By Lemma 7.1, each finite collection of pairwise crossing walls in $\widetilde{X}$ is either represented in a triangle $T$, or consists of several hyperplanes passing through a single cell. We will show that each such $T$ either lies in a periodic flat of type $(6,6,6),(4,8,8)$, or $(4,6,12)$, or all its sides have length $<B$ for some $B=B(X)$. The theorem then holds since there are only finitely many translates of cells, of small triangles, and of such periodic flat planes. We emphasize that since the periodic flat planes contain only finitely many parallelism classes of walls, our proof shows that there is an upper bound on the cardinality of a finite pairwise crossing collection, and hence there is no infinite pairwise crossing collection. Consequently every pairwise collection (is finite and) is represented within some cell, small triangle, or periodic flat. 
We will be applying results from Section 3 to reach conclusions about the even complex $\tilde{X}$. Recall that $\tilde{X}$ has a rhombus complex subdivision as discussed in Theorem 2.5. We will pass freely between the polygonal and rhombus viewpoints.

To show that a sufficiently large triangle $T$ lies within a periodic flat subcomplex $\widetilde{F}$, we will find $\widetilde{F}$ such that each side of $T$ intersects $\widetilde{F}$. Consequently, the three associated walls intersect within $\widetilde{F}$ by Theorem 3.12, and thus $T$ itself lies in $\widetilde{F}$ as they cannot cross elsewhere by Theorem 3.5 (2).

Assume that all three sides of $T$ are nontrivial. Let $D$ be a reduced disc diagram bounded by $T$. We first observe that for each wall $w$ within $D$, at least one 2-cell of $N(w)$ is not a square. Indeed, if $N(w)$ consists entirely of squares then it is convex. But then the walls associated to the two sides of $T$ that $w$ crosses, must actually cross within $N(w)$ by Theorem 3.12, but $N(w)$ does not contain the vertex of $T$ opposing it, which contradicts Theorem 3.5 (2).

As $D$ is reduced, each wall $w$ of $D$ crosses distinct sides of $T$ by Theorem 3.5.(2).

We now observe that there are boundedly many - say at most $E$ - exceptional walls $w$ in $D$ having the property that either $|w|<3 \lambda R$ or $\mathcal{N}_{R}(w)$ is not flat, where $R$ is the constant of Lemma 8.5 and $\lambda=\cot \left(\frac{\pi}{6}\right)$. Indeed, we will show that there are at most $E=3 f(3 \lambda R)+K Q$ such exceptional walls, by showing that the number of "short walls" is bounded by $3 f(3 \lambda R)$ and the number of "non-flat" walls is bounded by a constant $K Q$.

Lemma 8.3 shows that there are at most $3 f(3 \lambda R)$ walls in $D$ of length $\leq 3 \lambda R$. Indeed, any such wall must cross $T$ within $f(3 \lambda R)$ of one of the three corners.

Let $K=K(X)$ be an upper bound on the number of 1-cells (and hence walls) passing through an $R$-ball about a 0 -cell in $\widetilde{X}$. It follows that there are at most $K$ walls passing through an $R$-ball in $D$. Indeed, the walls in $D$ lift to distinct walls in $\widetilde{X}$, since if $w_{1}, w_{2}$ were walls of $D$ that lifted to the same wall in $\tilde{X}$, then some side of $T$ would be crossed by both $w_{1}$ and $w_{2}$. This contradicts our assumption that the sides of $T$ are geodesics by Theorem 3.5 (2). By Lemma 8.6, the number of negatively curved 0 -cells in $D$ is $\leq Q$. There are thus at most $K Q$ walls in $D$ whose $R$-neighborhoods are not flat.

We claim that $\mathcal{N}_{R}(w)$ is automatically "wide" when $w$ is non-exceptional, and consequently $\mathcal{N}_{R}(w)$ actually lies in a periodic flat by Lemma 8.5 . To see this claim, first observe that uniqueness of geodesics shows that $\mathcal{N}_{R}(w)$ has convex intersection with each geodesic side of $T$. Let $a, b, c$ denote the three corners of $T$, and let $A, B$, $C$ denote the sides of $T$ opposite them. The angle between a side of $T$ and a wall $w$ is $\geq \frac{\pi}{6}$. Indeed, the largest regular polygon that could occur with an open Euclidean neighborhood is a dodecagon. Consequently, there is an upper bound $\lambda R$ on the parts of $w$ along $\mathcal{N}_{R}(W) \cap C$ and $\mathcal{N}_{R}(W) \cap A$. The third side $B$ of $T$ can only intersect $\mathcal{N}_{R}(w)$ on the side of $w$ not containing $b$. Therefore a remaining portion of $\mathcal{N}_{R}(W)$ is isometric to $[0, R] \times[0,(3 \lambda R-2 \lambda R)]$.

Let $W_{a}$ denote the set of (non-exceptional) walls $w$ with the property that $w$ crosses $B, C$, and that $|w| \geq \lambda R$ and $\mathcal{N}_{R}(w)$ is flat. Define $\mathcal{W}_{b}$ and $\mathcal{W}_{c}$ similarly. 
If there are walls in two of $\mathcal{W}_{a}, \mathcal{W}_{b}, \mathcal{W}_{c}$ that cross each other, then the 2-cell where they cross has a flat open neighborhood in $D$. (Here we assume $R$ is chosen so that it also exceeds the radius of any polygon of $X$.) In particular, one of its bounding vertices has a flat open neighborhood and hence their associated periodic flats (of Lemma 8.5) are actually the same flat $\widetilde{F}$, which must then contain $T$ as above. We refer the reader to Figure 12. We will find such a crossing pair under the assumption that $T$ is sufficiently large as quantified below.
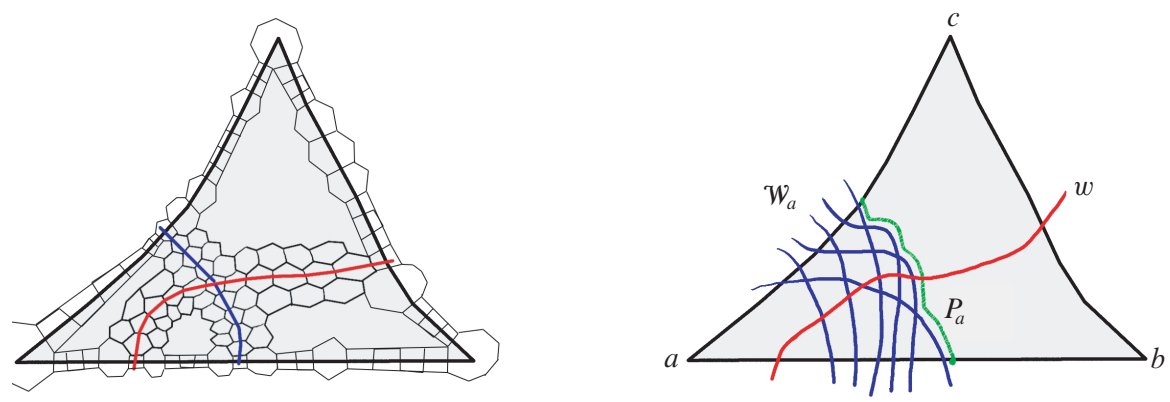

Figure 12. To see that a large wall-triangle $T$ resides in a periodic flat, we show as on the left, that there is a pair of intersecting flat strips that lie in the same periodic flat, and cross all three sides of $T$. The existence of the second strip in the pair follows an argument illustrated by the diagram on the right.

Let $U_{a}$ denote the union of the carriers of walls in $\mathcal{W}_{a}$. There is an outermost path $P_{a}$ on $\partial U_{a}$ that separates $a$ from $b, c$. In the degenerate case where $\mathcal{W}_{a}=\varnothing$ we define $P_{a}$ to be the trivial path at $a$. We define $P_{b}$ and $P_{c}$ analogously. If the endpoints of $P_{b}$ and $P_{a}$ on $C$ lie closer to $a$ and $b$ respectively, then there must be a wall in $\mathcal{W}_{b}$ that crosses a wall in $\mathcal{W}_{a}$, and we are done. The analogous statement holds for the other two sides of $T$. We shall therefore work under the assumption that $P_{a}, P_{b}, P_{c}$ cut off disjoint corners of $T$, in the sense that the endpoints of $P_{v}$ are closer to $v$ in the above sense.

We show below that when $T$ is sufficiently large, then at least one of $\left|P_{a}\right|,\left|P_{b}\right|$, $\left|P_{c}\right|$ exceeds the number $E$ of exceptional walls. Let us assume without loss of generality that $\left|P_{a}\right|>E$. It follows that there must be a 1-cell of $P_{a}$ that is dual to a non-exceptional wall $w$. If $w$ crosses both of $B, C$ then $w \in \mathcal{W}_{a}$, which is impossible by our choice of $P_{a}$. So let us assume that $w \in \mathcal{W}_{b}$ or $w \in \mathcal{W}_{c}$ and so $w \operatorname{crosses} A$. Finally, $w$ must cross a wall $w_{a} \in \mathcal{W}_{a}$ which itself crosses $B, C$, and we have found the desired pair of walls contained in the same periodic flat $\widetilde{F}$.

We now quantify how large $T$ must be to ensure that one of $\left|P_{a}\right|,\left|P_{b}\right|,\left|P_{c}\right|>E$ : Let $T_{a}$ denote the corner of $T$ subtended by $P_{a}$, and define $T_{b}$ and $T_{c}$ similarly. Let $|T|$ denote the number of edges in $T$, and let $\left|T_{a}\right|$ denote the number of edges in $T_{a}$ (which is the concatenation of two paths) and define $\left|T_{b}\right|$ and $\left|T_{c}\right|$ similarly. Observe that $|T| \leq\left|T_{a}\right|+\left|T_{b}\right|+\left|T_{c}\right|+2 E$. Suppose that $\left|P_{a}\right|,\left|P_{b}\right|,\left|P_{c}\right| \leq E$. 
Then arguing trigonometrically as in Lemmas 8.3 and 8.2, we have $\left|T_{a}\right|,\left|T_{b}\right|,\left|T_{c}\right| \leq$ $\left(\frac{1}{\sin \frac{\pi}{p}}+1\right) E$ where $p$ is an upper bound on the number of sides of each 2-cell of $X$. Thus $|T| \leq\left|T_{a}\right|+\left|T_{b}\right|+\left|T_{c}\right|+2 E \leq 3\left(\frac{1}{\sin \frac{\pi}{p}}+1\right) E+2 E$. Accordingly if $|T|>3\left(\frac{1}{\sin \frac{\pi}{p}}+1\right) E+2 E$ then at least one of $\left|P_{a}\right|,\left|P_{b}\right|,\left|P_{c}\right|>E$.

\section{Penrose tiling and related examples}

Penrose introduced a pair of polygons with matching rules called the "kite" and "dart" that can tile the plane but not periodically [Pen80]. Accompanying these are a related pair of rhombi with matching conditions illustrated in Figure 13. His aperiodic tilings have led to a flurry of continuous activity and study from various viewpoints. Any Penrose tiling of $\mathbb{E}^{2}$ by these rhombi is a simply-connected rhombus complex, whose dual cube complex is isomorphic to the standard cube complex of $\mathbb{E}^{5}$.

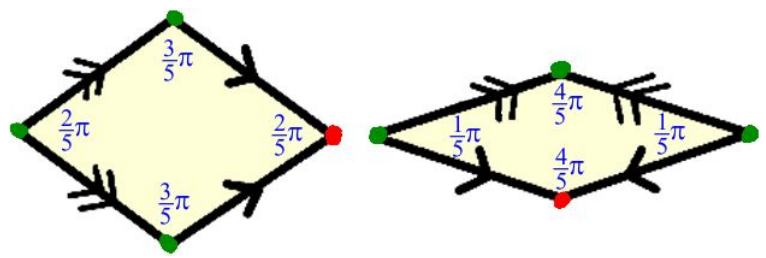

Figure 13. The Penrose complex $P$ is obtained by gluing the two rhombi above together.

In [ $\mathrm{dB} 81]$, de Bruijn had previously discovered this viewpoint on the Penrose rhombus tiling of $\mathbb{E}^{2}$ and it led him to beautiful explanations of the construction and behavior of the Penrose tilings, as well as to the production of many further examples. He calls the carriers of walls ribbons, and shows how the ribbons are intimately related to the structure of the tilings. He also recognized that the tilings are intrinsically related to a 2-dimensional plane cutting diagonally through the usual cubical tiling of $\mathbb{E}^{5}$. From our viewpoint, this is the natural embedding of a graph that is a wallspace in its dual CAT(0) cube complex! Using his viewpoint, de Bruijn is able to produce many other types of Penrose-like rhombus tilings, coming from other diagonal embeddings of $\mathbb{E}^{2}$ in $\mathbb{E}^{d}$ for various $d$.

Example 9.1 (Penrose complex). The Penrose complex $P$ is the complex obtained by identifying the 1-cells of the two rhombi in Figure 13 according to the labels. We note that it has two 0-cells, two 1-cells and two 2-cells.

While $P$ is not itself nonpositively curved, it is tempting to produce nonpositively curved rhombus complexes $X$ that admit a combinatorial map $X \rightarrow P$ which is a near-immersion in the sense that it is an immersion (i.e. local injection) except perhaps at the 0 -cells. Since $\pi_{1}\left(P-P^{0}\right)$ is free, a simple way to produce numerous 
compact near-immersions $X \rightarrow P$ is through branched-covering spaces where the branching takes place at the 0 -cells. When the induced maps $\operatorname{link}(x) \rightarrow \operatorname{link}(p)$ are "large" enough covering spaces, the angled 2-complex $X$ will be nonpositively curved.

In this way we are led to produce infinitely many compact rhombus complexes $X$ with the property that $\pi_{1} X$ cannot contain a $\mathbb{Z}^{2}$ subgroup. Indeed, the flat torus theorem would then yield a periodic flat plane in $\widetilde{X}$, and this would map to a periodic flat plane immersed in $P$ - which is impossible.

We are thus left to ask the question: Is there a nonpositively curved complex $X$ that admits a near-immersion to the Penrose complex, such that $\tilde{X}$ contains a (necessarily aperiodic) flat? The following shows that there is no such example.
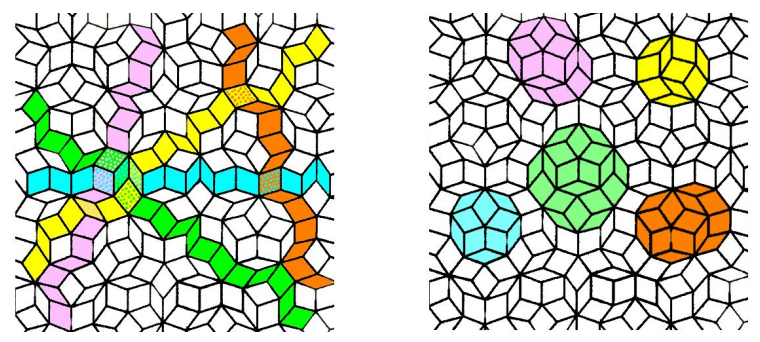

Figure 14. On the left are five distinctly sloped de Bruijn ribbons - these are carriers of walls in our viewpoint. On the right are typical maximal convex proper subcomplexes.

Theorem 9.2. Let $X$ be a compact nonpositively curved complex that admits an immersion to the Penrose tiling complex. Then $\pi_{1} X$ is word-hyperbolic.

Proof. We first show that any convex proper subcomplex of a flat $F$ in $\tilde{X}$ has uniformly bounded diameter. Indeed, the cell structure on $F$ is a Penrose tiling. And the boundary path of a convex subcomplex $S$ has at most two consecutive edges without turning, and each turn has angle $\leq \pi-\frac{\pi}{5}$. Since the sum of the defects is $2 \pi$ there can be at most 10 corners.

It follows that $\tilde{X}$ has isolated flats. Consequently, from Proposition 6.2, we see that each flat is periodic. But no flat can be periodic - as this is the remarkable property of the Penrose tiles.

Acknowledgement. We are grateful to the referee for corrections that improved the exposition. 


\section{References}

[BH99] M. R. Bridson and A. Haefliger, Metric spaces of non-positive curvature. Grundlehren Math. Wiss. 319, Springer-Verlag, Berlin 1999. Zbl 0988.53001 MR 1744486

[dB81] N. G. de Bruijn, Algebraic theory of Penrose's non-periodic tilings of the plane. I, II. Nederl. Akad. Wetensch. Indag. Math. 43 (1981), 39-52, 53-66. Zbl 0457.05021 MR 609465 Zbl 0457.05022

[GS90] S. M. Gersten and H. B. Short, Small cancellation theory and automatic groups. Invent. Math. 102 (1990), 305-334. Zbl 0714.20016 MR 1074477

[HP98] F. Haglund and F. Paulin, Simplicité de groupes d'automorphismes d'espaces à courbure négative. In The Epstein Birthday schrift, Geom. Topol. Monogr. 1, University of Warwick, Institute of Mathematics, Coventry 1998, 181-248. Zbl 0916.51019 MR 1668359

[Hru04] G. C. Hruska, Nonpositively curved 2-complexes with isolated flats. Geom. Topol. 8 (2004), 205-275. Zbl 1063.20048 MR 2033482

[HW] G. C. Hruska and D. T. Wise, Finiteness properties of cubulated groups. Compositio Math., to appear.

[MW02] J. P. McCammond and D. T. Wise, Fans and ladders in small cancellation theory. Proc. London Math. Soc. (3) 84 (2002), 599-644. Zbl 1022.20012 MR 1888425

[NR98] G. A. Niblo and L. D. Reeves, The geometry of cube complexes and the complexity of their fundamental groups. Topology 37 (1998), 621-633. Zbl 0911.57002 MR 1604899

[NR03] G. A. Niblo and L. D. Reeves, Coxeter groups act on CAT(0) cube complexes. $J$. Group Theory 6 (2003), 399-413. Zbl 1068.20040 MR 1983376

[Pen80] R. Penrose, Pentaplexity: a class of nonperiodic tilings of the plane. Math. Intelligencer 2 (1979/80), 32-37. Zbl 0426.52005 MR 558670

[Sag95] M. Sageev, Ends of group pairs and non-positively curved cube complexes. Proc. London Math. Soc. (3) 71 (1995), 585-617. Zbl 0861.20041 MR 1347406

[Sag97] vM. Sageev, Codimension-1 subgroups and splittings of groups. J. Algebra 189 (1997), 377-389. Zbl 0873.20028 MR 1438181

[Sta91] J. R. Stallings, Non-positively curved triangles of groups. In Group theory from a geometrical viewpoint (Trieste, 1990), World Sci. Publ., Singapore 1991, 491-503. Zbl 0843.20033 MR 1170374

[Wis96] D. T. Wise, Non-positively curved squared complexes: Aperiodic tilings and nonresidually finite groups. Ph.D. thesis, Princeton University, Princeton 1996. Available at http://www.math.mcgill.ca/wise/papers.html

[Wis04] D. T. Wise, Cubulating small cancellation groups. Geom. Funct. Anal. 14 (2004), 150-214. Zbl 1071.20038 MR 2053602

[Wis05] D. T. Wise, Approximating flats by periodic flats in CAT(0) square complexes. Canad. J. Math. 57 (2005), 416-448. Zbl 1085.20027 MR 2124924 
Received May 28, 2011; revised November 18, 2011

D. Janzen, Mathematics, John Abbott College, Ste Anne de Bellevue, Quebec, Canada H9X 3L9

E-mail: david.janzen@johnabbott.qc.ca

D. T. Wise, Department of Mathematics \& Statistics, McGill University, Montreal, Quebec, Canada H3A 2K6

E-mail: wise@math.mcgill.ca 